\title{
BMJ Open Emergence agitation during recovery from intracranial surgery under general anaesthesia: a protocol and statistical analysis plan for a prospective multicentre cohort study
}

Li-Mei Yan, ${ }^{1,2}$ Han Chen, ${ }^{1,3}$ Rong-Guo Yu, ${ }^{3}$ Zhu-Heng Wang, ${ }^{1,4}$ Guan-Hua Zhou, ${ }^{4}$ Yong-Jin Wang, ${ }^{5}$ Xia Zhang, ${ }^{5}$ Ming Xu, ${ }^{1}$ Lu Chen, ${ }^{1,6}$ Jian-Xin Zhou, ${ }^{1}$ on behalf of the Acute Brain Injury and Critical Care Research Collaboration (ABC Research Collaboration)

To cite: Yan L-M, Chen $\mathrm{H}$, Yu R-G, et al. Emergence agitation during recovery from intracranial surgery under general anaesthesia: a protocol and statistical analysis plan for a prospective multicentre cohort study. BMJ Open 2015:5:e007542.

doi:10.1136/bmjopen-2014007542

- Prepublication history for this paper is available online. To view these files please visit the journal online (http://dx.doi.org/10.1136/ bmjopen-2014-007542).

Received 25 December 2014 Revised 24 March 2015 Accepted 27 March 2015

CrossMark

For numbered affiliations see end of article.

Correspondence to Professor Jian-Xin Zhou; zhoujx.cn@gmail.com

\section{ABSTRACT}

Introduction: Emergence agitation after intracranial surgery is an important clinical issue during anaesthesia recovery. The aim of this multicentre cohort study is to investigate the incidence of emergence agitation, identify the risk factors and determine clinical outcomes in adult patients after intracranial surgery under general anaesthesia. Additionally, we will deliberately clarify the relationship between postoperative pneumocephalus and agitation.

Methods and analysis: The present study is a prospective multicentre cohort study. Five intensive care units (ICUs) in China will participate in the study. Consecutive adult patients admitted to the ICUs after intracranial surgery will be enrolled. SedationAgitation Scale (SAS) or Richmond Agitation-Sedation Scale (RASS) will be used to evaluate the patients $12 \mathrm{~h}$ after the enrolment. Agitation is defined as an SAS score of $5-7$, or an RASS score of +2 to +4 . According to the maximal SAS and RASS score, patients will be divided into two cohorts: the agitation group and the non-agitation group. Factors potentially related to emergence agitation will be collected at study entry, during anaesthesia and operation, during postoperative care. Univariate analyses between the agitation and the non-agitation groups will be performed. The stepwise backward logistic regression will be carried out to identify the independent predictors of agitation. Patients will be followed up for $72 \mathrm{~h}$ after the operation. Accidental self-extubation of the endotracheal tube and removal of other catheters will be documented. The use of sedatives and analgesics will be collected.

Ethics and dissemination: Ethics approval has been obtained from each of five participating hospitals. Study findings will be disseminated through peer-reviewed publications and conference presentations.

Trial registration number: NCT02318199.
Strengths and limitations of this study

Patients after intracranial operations are more vulnerable to the stress resulting from emergence agitation during the recovery from general anaesthesia. However, the precise incidence and risk factors of agitation have not been fully investigated in this subset of patients. The main strength of our study is that we will provide the evidence of incidence and risk factors of agitation in a large sample sized multicentre cohort study.

- Pain has been identified as an independent risk factor for emergence agitation in nonneurosurgical patients. Patient's self-report pain scales, such as the visual analogue scale or verbal numerical rating scale, have been recommended in pain evaluation in critically ill patients. However, evaluations of self-reported pain scales require a patient's ability to communicate. Consciousness impairment due to intracranial manipulation and postoperative sedation may influence the reliability of these evaluations. For these reasons, we do not incorporate the evaluations of self-report pain scales into our clinical practice, and only document the patient's symptom of pain. This is the main limitation of the study.

- The sample size calculation is based on our previous study with a relatively high incidence of agitation. It is likely that the incidence will be lower in this multicentre study. We could not set an anticipated incidence because of the lack of such information. However, this may decrease the power of this study.

\section{INTRODUCTION}

Emergence agitation is a significant clinical issue during recovery from general anaesthesia. ${ }^{1}$ Emergence agitation can suddenly become 
dangerous and have serious consequences, such as selfextubation, accidental removal of catheters and injury. Patients after intracranial operations are more vulnerable to the stress resulting from emergence agitation during the recovery from general anaesthesia. ${ }^{2}{ }^{3}$ In our previous pilot study with 123 cases, ${ }^{4}$ we found that the incidence of agitation was $29 \%$ in patients admitted to the intensive care unit (ICU) after intracranial operations for brain tumours, and this incidence was higher than in those undergoing no intracranial operations. ${ }^{5-8}$ Observational investigations in patients after intracranial surgeries have been scarce. Three large cohort studies that investigated emergence agitation after general anaesthesia excluded neurosurgical patients. ${ }^{6-}$ ${ }^{8}$ Several randomised controlled trials, which compared the influence of different anaesthetics on emergence agitation in patients after intracranial surgeries, revealed that the incidence of agitation during the early postoperative period varied from $2.5 \%$ to $13.3 \% .^{9-11}$ In a Europe multicentre randomised controlled trial, 411 patients were enrolled after elective supratentorial intracranial surgery under general anaesthesia, and emergence agitation was compared among three different anaesthesia maintenance methods (sevoflurane-remifentanil, sevoflurane-fentanyl and propofol-remifentanil). ${ }^{11}$ No significant difference was found in the incidence of agitation among the three groups $(3.7 \%, 5.2 \%$ and $6.5 \%) .{ }^{11}$ The incidence of agitation reported in our pilot study was still much higher than these results. ${ }^{4-11}$ These discrepancies warrant a prospective cohort study with a large sample size to determine the precise incidence of emergence agitation in patients after intracranial surgery.

The causes of agitation are multifactorial. Studies in a non-neurosurgical population have found that pain, endotracheal intubation, duration of surgery and history of long-term treatment with antidepressant agents were the most common independent risk factors for emergence agitation. ${ }^{6-8}$ In our pilot study, we identified two specified independent predictors for emergence agitation after craniotomy under general anaesthesia, prolonged balanced anaesthesia and intracranial operation via the frontal lobe approach. ${ }^{4}$ These results indicate that we should pay more attention to this subset of patients. During the performance of our pilot study, we noticed that pneumocephalus after craniotomy might be a potential risk factor for emergence agitation. However, we did not deliberately collect this information because of the lack of design in protocol. Pneumocephalus might be preventable if neurosurgeons are more cautious during the suture of the dura mater. Further studies are needed to clarify whether pneumocephalus would be a risk factor for agitation.

In this prospective multicentre cohort study, we will prospectively enrol adult patients after intracranial surgery under general anaesthesia, investigate the incidence of emergence agitation, identify the risk factors and determine clinical outcomes. Special attention will be paid to clarify the relationship between postoperative pneumocephalus and agitation. These results could provide basic data for the prevention and treatment of emergence agitation in postoperative neurosurgical patients.

\section{METHODS AND ANALYSIS}

\section{Study design overview}

This study is a prospective multicentre cohort study in patients after intracranial surgery under general anaesthesia.

\section{Study setting and population}

Five ICUs in China will take part in this multicentre cohort study. They are:

1. The Department of Critical Care Medicine (20 beds), Beijing Tiantan Hospital (1100 beds), Capital Medical University, Beijing. The nurse-to-bed ratio in the ICU is $3: 1$.

2. The Department of Critical Care Medicine (18 beds), Inner Mongolia People's Hospital (2315 beds), Hohhot, Inner Mongolia. The nurse-to-bed ratio in the ICU is 2.5:1.

3. The Surgical Intensive Care Unit (22 beds), Fujian Provincial Clinical College (2500 beds), Fujian Medical University, Fuzhou, Fujian. The nurse-to-bed ratio in the ICU is $3: 1$.

4. The Department of Critical Care Medicine (22 beds), Daxing Teaching Hospital (1080 beds), Capital Medical University, Beijing. The nurse-to-bed ratio in the ICU is 2.5:1.

5. The Department of Critical Care Medicine (14 beds), Bethune International Peace Hospital (1500 beds), Hebei Medical University, Shijiazhuang, Hebei. The nurse-to-bed ratio in the ICU is 2.5:1.

Each of the participating ICUs is managed by full-time intensive care physicians with a $24 \mathrm{~h} / 7$ days on-duty senior medical staff.

Consecutive patients admitted to the ICUs after intracranial surgery are screened daily for study eligibility. The inclusion criterion is adult patients after intracranial operations. The exclusion criteria include:

1. Age younger than 18 years, or older than 80 years;

2. Transsphenoidal surgery, cerebrospinal fluid (CSF) shunt and CSF drainage surgery;

3. Unarousable state during the first $24 \mathrm{~h}$ after the operation. Unarousable state is defined as a motor response to external stimuli in Glasgow Coma Scale (GCS) lower than $5 ;^{12}$

4. Preoperative impairment of consciousness, evaluated by a medical document;

5. Pregnant or lactating women;

6. Interval longer than $24 \mathrm{~h}$ between the end of the surgery and ICU admission;

7. Enrolled in another trial.

Patients were enrolled only once unless they were discharged from the hospital and readmitted more than 180 days after the first enrolment. 


\section{End point measures}

The primary end point will be the incidence and risk factors of emergence agitation during the first $12 \mathrm{~h}$ after an intracranial operation. The levels of agitation and sedation will be evaluated by a Sedation-Agitation Scale $(\mathrm{SAS})^{13}$ or a Richmond Agitation-Sedation Scale (RASS). ${ }^{14}$ Agitation is defined as an SAS score of $5-7$, or an RASS score of +2 to +4 . Risk factors for emergence agitation will be investigated. Additionally, we will particularly investigate the relationship between postoperative pneumocephalus and agitation.

The secondary end points will be the clinical outcome variables, which include self-extubation of the endotracheal tube and accidental removal of the central venous or bladder catheters, unexpected reoperation within $72 \mathrm{~h}$ after surgery and ICU discharge at postoperative day 1 . The uses of sedatives and analgesics during the ICU stay are also documented.

\section{Routine practice of anaesthesia and postoperative care}

During the study, routine practices of anaesthesia and postoperative care are followed, and no attempts will be made to change or influence the standard of care. Before organising this multicentre study, we sent inquiry letters to the ICU directors in 13 hospitals in China. All are members of the Acute Brain Injury and Critical Care Research Collaboration (ABC Research Collaboration). The questions were:

1. Are intracranial surgeries performed under general anaesthesia in your hospital?

2. Are patients after intracranial surgeries routinely admitted to the ICU for postoperative care?

3. What are the most commonly used anaesthetics (intravenous and/or inhalational) and analgesics in your hospital?

4. Is evaluation of GCS and SAS or RASS incorporated into your routine practice for postoperative care?

5. Are sedatives used in the treatment of agitation? If yes, what is the most common agent?

Five ICUs will participate in this multicentre study because routine practices of anaesthesia and postoperative care for patients undergoing intracranial surgeries are similar in these ICUs.

All intracranial operations are performed under general balanced anaesthesia or total intravenous anaesthesia in participating hospitals. Typically, anaesthesia is induced with intravenous propofol and fentanyl or sufentanil. Tracheal intubation is facilitated with intravenous vecuronium or rocuronium. Anaesthesia is maintained with propofol and/or sevoflurane or isoflurane. Fentanyl or sufentanil is administered intermittently, as needed. Vecuronium or rocuronium is administered according to train-of-four monitoring. The choice of agents is at the discretion of the anaesthesiologist. ${ }^{15}$

As long as there are enough beds, patients after craniotomy are routinely transferred to the ICU. In the ICU, neurological examinations including GCS, focal signs and pupillary examination are performed by nurses hourly or as needed. Haemodynamic and respiratory monitoring includes a five-lead continuous ECG, a pulse oximeter, non-invasive blood pressure and capnography. A postoperative CT scan is not routinely used, but is usually performed in patients who exhibit unexplained delayed awakening or new neurological deficits during the first $24 \mathrm{~h}$ postoperatively. Patients are discharged from the ICU the next morning once they achieve normal neurological, haemodynamic and respiratory statuses.

The level of agitation and sedation is evaluated by SAS or RASS. At least one of these two scales has been incorporated into clinical practice in all five hospitals more than 1 year before the enrolment of the patients. All of the physicians and nurses in the ICU have been trained. Nurses assess and document each patient's SAS or RASS every $4 \mathrm{~h}$ or as needed. In patients with agitation (SAS $=5-7$ or RASS $=+2$ to +4 ), both the nurse and physician perform careful evaluation to identify the possible organic causes of agitation, such as acute deterioration of respiratory and circulatory status, a new neurological event, pain and hypoglycaemia. Fentanyl $(25 \mu \mathrm{g})$ is administered intravenously on an as-needed basis. Midazolam or propofol is used, and the level of sedation is titrated to an SAS of 3-4 (or an RASS of -2 to +1 ).

\section{Definition and documentation of agitation}

Agitation is defined as an SAS score of 5-7, or an RASS score of +2 to +4 . During the study period, two investigators in each centre evaluate and document the SAS or RASS scores of the enrolled patients on an hourly basis. These investigators are the chief nurses who do not participate in the care of the enrolled patients. Two other investigators review the nursing records daily. Finally, the maximal SAS or RASS for each patient will be determined and confirmed by the four investigators in a daily meeting.

SAS or RASS will be evaluated for $12 \mathrm{~h}$ after the enrolment of the patient. According to the maximal SAS or RASS score, patients will be divided into two cohorts: the agitation group and the non-agitation group.

\section{Data collection}

During the study period, the number of intracranial surgeries and the number of ICU admissions will be registered. The reason(s) for patients who directly return to the neurosurgical ward will be documented.

For each patient, the following data are collected at study entry: demographic characteristics (sex, age, height and body weight), history of smoking and alcohol abuse, long-term ( $>1$ month) use of antidepressant drugs or benzodiazepines, diagnosis and location of the lesion, and American Society of Anesthesiologists (ASA) physical status. Data collected during the anaesthesia and operation include: approach of the operation, duration of anaesthesia, amount of bleeding, fluid balance and method of general anaesthesia. The postoperative data collected include: GCS at ICU admission, presence 
of endotracheal intubation, need for mechanical ventilation, presence of an external ventricular drainage tube, symptoms of pain, episodes of pulse oxygen saturation $\left(\mathrm{SpO}_{2}\right)$ less than $90 \%$, respiratory rate less than 8 breaths/min, mean blood pressure greater than $130 \mathrm{~mm} \mathrm{Hg}$ or less than $70 \mathrm{~mm} \mathrm{Hg}$, and blood glucose greater than $10 \mathrm{mmol} / \mathrm{L}$.

In patients with a postoperative CT scan, pneumocephalus, brain swelling, intracranial haemorrhage and ischaemia or infarction will be documented. Pneumocephalus is defined as intracranial gaseous volume greater than $30 \mathrm{~cm}^{3}$, according to the following simple formula recommended by Broderick et al: ${ }^{16}$

\section{Intracranial gaseous volume $\left(\mathrm{cm}^{3}\right)=\mathrm{ABC} / 2$}

where A represents the maximum diameter of gas accumulation; $\mathrm{B}$ represents the maximum diameter perpendicular to A on an axial slice showing maximal gas accumulation; $\mathrm{C}$ represents the numbers of $1 \mathrm{~cm}$ thick CT slices on which the gaseous body is visualised. There are two methods for the evaluation of pneumocephalus volume: the subjective category $^{17}$ and the volume calculation by the formula aforementioned. ${ }^{18}$ The volume calculation method will be used in this study because we think that this method will provide an objective quantitative evaluation of the pneumocephalus.

Patients will be followed up for $72 \mathrm{~h}$ after surgery. The primary clinical outcome variables include selfextubation of the endotracheal tube and accidental removal of central venous or bladder catheters. Secondary clinical outcome variables include unexpected reoperation within $72 \mathrm{~h}$ after surgery and ICU discharge at postoperative day 1 . The uses of sedatives and analgesics during the ICU stay are also documented.

\section{Current sample size justification}

In our previous pilot study, we found that the incidence of emergence agitation was $29 \%$ and identified five risk factors. ${ }^{4}$ We also found a potential association between pneumocephalus and postoperative agitation in a secondary analysis (unpublished), which was based on limited data and lack of power to test the hypothesis. We therefore propose that this study examine the relationship between these two binary variables in a similar cohort and use the previous data to estimate the sample size requirement.

The proposed study will retain $95 \%$ power and conduct two-sided tests with a significance level of 0.05 . The computation is based on the relationship between pneumocephalus and post-operative agitation. The OR between these two factors is calculated with pneumocephalus as the binary covariate and agitation as the binary dependent variable. We then adjust the sample size requirement for a multiple logistic regression by a variance inflation factor with the presence of other covariates (five risk factors which were previously identified). ${ }^{19}$ Since only a small fraction of enrolled patients may receive a CT scan examination within $12 \mathrm{~h}$ after neurosurgical operations in our routine practice, we estimate that approximately $25 \%$ of enrolled patients would be available for evaluation of pneumocephalus via a CT scan. In other words, the total sample size required for enrolment should be four times more than the valid sample size required for a multiple logistic regression analysis. We anticipate that the proportion of enrolled patients who received a CT scan with missing primary evaluation will be small $(<2 \%)$. Therefore, we may expect that the sample size of 400 patients will provide the study with $95 \%$ power to detect a difference in primary end point at $5 \%$ of significance.

Since the volume of intracranial operations in Beijing Tiantan Hospital is nearly four times that of the other four hospitals, we set the maximal recruitment of 200 patients in Beijing Tiantan Hospital and 50 in each of the other four hospitals.

\section{Statistical analysis}

Statistical analyses are performed using MS Excel for Mac (Microsoft Corporation, Beijing, China) and SPSS statistical software (V.20.0, SPSS, Chicago, Illinois, USA). Categorical variables are expressed as percentages. Continuous data are checked for normal distribution by the Kolmogorov-Smirnov test and are reported as the means and SDs or the medians with 25th and 75th percentiles, when applicable.

The distribution of maximal SAS score is analysed, and the incidence of agitation is calculated to present the epidemiological characteristics. Univariate analyses between the agitation and non-agitation groups are performed. Categorical variables are analysed by the $\chi^{2}$ test. Comparisons of continuous data are performed by using the unpaired $t$ test for normally distributed variables and the Mann-Whitney U test for non-normally distributed variables. Factors with $p$ values $<0.05$ are included in multivariate analysis (stepwise backward logistic regression) to identify independent predictors of agitation. ORs and their $95 \%$ CIs are used to assess the independent contributions of significant factors. The HosmerLemeshow test is used to determine the appropriateness of the model.

A p value of less than 0.05 will be considered statistically significant.

\section{Data quality and training of research staff}

A streamlined data case report form will be used in data collection. All principal investigators, research coordinators, physicians and senior nurses at each participating centre are trained on study procedures, including data collection and bedside training on the performance of GCS, SAS and RASS evaluations. CT scans will be saved as Tagged Image File Format (TIFF) imaging for offline analysis. Diagnosis of brain swelling, intracranial haemorrhage and ischaemia or infarction, and calculation of pneumocephalus volume will be carried out by two senior physicians in the Department of Radiology in 
Beijing Tiantan Hospital blinded to the patient's condition of agitation.

\section{Interim revision of the protocol}

After receiving the reviewers' comments from BMJ Open, we held a meeting with the principal investigators in each participating centre and discussed the protocol systematically. The protocol was then revised, and thereafter four kinds of data will be collected prospectively as follows: (1) potential surgical factors with agitation, which include the position of surgery (sitting or not), the opening of the basal cistern during the operation (yes or no), closure of the dura mater (yes or no), and the amount of mannitol infusion during the operation; (2) the relationship of agitation with endotracheal extubation (time of extubation will be documented); (3) the convulsive seizure during the study period (defined as a transient episode of abnormal involuntary motor movements according to the definitions proposed by the International League Against Epilepsy and the International Bureau for Epilepsy). ${ }^{20}$ The performance of the EEG is at the discretion of the local clinical practice; and (4) the Glasgow Outcome Scale at hospital discharge. ${ }^{21}$ Approximately 200 patients had been enrolled by the time of the protocol revision (20 March 2015). For these already enrolled patients, the anaesthesia records, ICU nursing sheets and medical records will be reviewed retrospectively. These data will be reported in the final version of the results.

\section{ETHICS AND DISSEMINATION \\ Ethical considerations}

As no attempts will be made to change or influence standard clinical practices, informed consent was waived with IRB approval. Each participating hospital's IRB also approved this study with a waiver of informed consent.

\section{Dissemination plan}

Results of the trial will be submitted to an international peer-reviewed journal. Results will also be presented at national and international conferences relevant to the subject fields.

\section{TRIAL STATUS}

The study was initiated in January 2015, and will be completed in May 2015.

\section{SUMIMARY}

Emergence agitation can suddenly become dangerous and have serious consequences. Patients after intracranial operations are more vulnerable to the stress resulting from emergence agitation during the recovery from general anaesthesia. ${ }^{1-3}$ However, only a few studies have been performed to investigate the emergence agitation in neurosurgical patients. ${ }^{4-11}$ Our pilot study found that the incidence of agitation in ICU admitted patients after intracranial operations for brain tumours was higher than previously reported. ${ }^{4}$ We also identified two specified independent predictors for emergence agitation after craniotomy under general anaesthesia, prolonged balanced anaesthesia and intracranial operation via a frontal approach. ${ }^{4}$ These discrepancies and lack of evidence warrant a prospective cohort study with large sample size to determine the precise incidence and risk factors for emergence agitation in patients after intracranial surgery.

In this prospective multicentre cohort study, we will prospectively enrol adult patients after intracranial surgery under general anaesthesia, investigate the incidence of emergence agitation, identify the risk factors and determine clinical outcomes. We will pay special attention to investigate the relationship between postoperative pneumocephalus and agitation. These results could provide basic data for the prevention and treatment of emergence agitation in postoperative neurosurgical patients.

\section{Author affiliations}

${ }^{1}$ Department of Critical Care Medicine, Beijing Tiantan Hospital, Capital Medical University, Beijing, China

${ }^{2}$ Department of Critical Care Medicine, Inner Mongolia People's Hospital, Hohhot, Inner Mongolia, China

${ }^{3}$ Surgical Intensive Care Unit, Fujian Provincial Clinical College, Fujian Medical University, Fuzhou, Fujian, China

${ }^{4}$ Department of Critical Care Medicine, Daxing Teaching Hospital, Capital Medical University, Beijing, China

${ }^{5}$ Department of Critical Care Medicine, Bethune International Peace Hospital, Hebei Medical University, Shijiazhuang, Hebei, China

${ }^{6}$ Interdepartmental Division of Critical Care Medicine, University of Toronto, Toronto, Ontario, Canada

Acknowledgements The authors would like to thank Dr Zehui Tan, MD (Department of Internal Medicine, Sharp Rees-Stealy Medical Group, San Diego, California, USA) for her excellent advice on English writing.

Contributors L-MY, LC and J-XZ led the study concept and design. L-MY, $\mathrm{R}-\mathrm{GY}, \mathrm{G}-\mathrm{HZ}, \mathrm{Y}-\mathrm{JW}$ and $\mathrm{J}-\mathrm{XZ}$ are the principal investigators of the participating hospitals. All the authors were involved in contributing to the design of the work and the writing of the manuscript. L-MY wrote the first draft of the manuscript. All the authors provided critical review, and have given final approval of the final submitted manuscript.

Funding The study was funded by the Beijing Municipal Administration of Hospital (grant number ZYLX201502).

Competing interests None declared.

Ethics approval The Institutional Review Board (IRB) of Beijing Tiantan Hospital, Capital Medical University (KY2014-034-01), Inner Mongolia People's Hospital (\#20141218), Fujian Provincial Clinical College, Fujian Medical University (K2014-14-001), Daxing Teaching Hospital, Capital Medical University (201403018) and Bethune International Peace Hospital, Hebei Medical University (2014-No:12-1).

Provenance and peer review Not commissioned; externally peer reviewed.

Open Access This is an Open Access article distributed in accordance with the Creative Commons Attribution Non Commercial (CC BY-NC 4.0) license, which permits others to distribute, remix, adapt, build upon this work noncommercially, and license their derivative works on different terms, provided the original work is properly cited and the use is non-commercial. See: http:// creativecommons.org/licenses/by-nc/4.0/ 


\section{REFERENCES}

1. Whitlock EL, Vannucci A, Avidan MS. Postoperative delirium. Minerva Anestesiol 2011;77:448-56.

2. Bruder N, Ravussin P. Recovery from anesthesia and postoperative extubation of neurosurgical patients: a review. J Neurosurg Anesthesiol 1999;11:282-93.

3. Bruder NJ. Awakening management after neurosurgery for intracranial tumours. Curr Opin Anaesthesiol 2002;15:477-82.

4. Chen L, Xu M, Li GY, et al. Incidence, risk factors and consequences of emergence agitation in adult patients after elective craniotomy for brain tumor: a prospective cohort study. PLOS ONE 2014;9:e114239.

5. Rose DK. Recovery room problems or problems in the PACU. Can J Anaesth 1996;43:R116-28.

6. Lepouse C, Lautner CA, Liu L, et al. Emergence delirium in adults in the post-anaesthesia care unit. Br J Anaesth 2006;96: 747-53.

7. Radtke FM, Franck M, Hagemann L, et al. Risk factors for inadequate emergence after anesthesia: emergence delirium and hypoactive emergence. Minerva Anestesiol 2010;76:394-403.

8. Yu D, Chai W, Sun X, et al. Emergence agitation in adults: risk factors in 2,000 patients. Can J Anaesth 2010;57:843-88.

9. Todd MM, Warner DS, Sokoll MD, et al. A prospective, comparative trial of three anesthetics for elective supratentorial craniotomy. Propofol/fentanyl, isoflurane/nitrous oxide, and fentanyl/nitrous oxide. Anesthesiology 1993;78:1005-20.

10. Kanaya N, Kuroda H, Nakayama M, et al. Does propofol anesthesia increase agitation in neurosurgical patients?-a pilot study. Can $J$ Anaesth 2002;49:638-9.

11. Citerio G, Pesenti A, Latini R, et al. A multicentre, randomised, open-label, controlled trial evaluating equivalence of inhalational and intravenous anaesthesia during elective craniotomy. Eur $\mathrm{J}$ Anaesthesiol 2012;29:371-9.

12. Majerus S, Gill-Thwaites $\mathrm{H}$, Andrews $\mathrm{K}$, et al. Behavioral evaluation of consciousness in severe brain damage. Prog Brain Res 2005;150:397-413.

13. Riker RR, Picard JT, Fraser GL. Prospective evaluation of the Sedation-Agitation Scale for adult critically ill patients. Crit Care Med 1999;27:1325-9.

14. Sessler CN, Gosnell MS, Grap MJ, et al. The Richmond Agitation-Sedation Scale: validity and reliability in adult intensive care unit patients. Am J Respir Crit Care Med 2002;166:1338-44.

15. Cai YH, Zeng HY, Shi ZH, et al. Factors influencing delayed extubation after infratentorial craniotomy for tumour resection: a prospective cohort study of 800 patients in a Chinese neurosurgical centre. J Int Med Res 2013;41:208-17.

16. Broderick JP, Brott TG, Grotta JC. Intracranial hemorrhage volume measurement. Stroke 1994;25:1081.

17. Reasoner DK, Todd MM, Scamman FL, et al. The incidence of pneumocephalus after supratentorial craniotomy. Observations on the disappearance of intracranial air. Anesthesiology 1994;80:1008-12.

18. Beppu T, Ogasawara K, Ogawa A. Alleviation of intracranial air using carbon dioxide gas during intraventricular tumor resection. Clin Neurol Neurosurg 2006;108:655-60.

19. Hsieh FY, Bloch DA, Larsen MD. A simple method of sample size calculation for linear and logistic regression. Stat Med 1998;17:1623-34

20. Fisher RS, van Emode Boas W, Blume W, et al. Epileptic seizures and epilepsy: definitions proposed by the International League Against Epilepsy (ILAE) and the International Bureau for Epilepsy (IBE). Eilepsia 2005;46:470-2.

21. Jennett $B$, Bond M. Assessment of outcome after severe brain damage. Lancet 1975;1:480-4. 